\title{
Overview of the CHarring Ablator Response (CHAR) Code
}

\author{
Adam J. Amar,* A. Brandon Oliver, ${ }^{*}$ Benjamin S. Kirk, ${ }^{*}$ and Giovanni Salazar* \\ NASA Lyndon B. Johnson Space Center \\ 2101 NASA Parkway, Houston, TX, 77058 \\ Justin Droba ${ }^{\dagger}$ \\ HX5
}

2101 NASA Parkway, Houston, TX, 77058

\begin{abstract}
An overview of the capabilities of the $C H$ arring $A$ blator Response (CHAR) code is presented. $C H A R$ is a one-, two-, and three-dimensional unstructured continuous Galerkin finite-element heat conduction and ablation solver with both direct and inverse modes. Additonally, $C H A R$ includes a coupled linear thermoelastic solver for determination of internal stresses induced from the temperature field and surface loading. Background on the development process, governing equations, material models, discretization techniques, and numerical methods is provided. Special focus is put on the available boundary conditions including thermochemical ablation, surface-to-surface radiation exchange, and flowfield coupling. Finally, a discussion of ongoing development efforts is presented.
\end{abstract}

\section{Nomenclature}

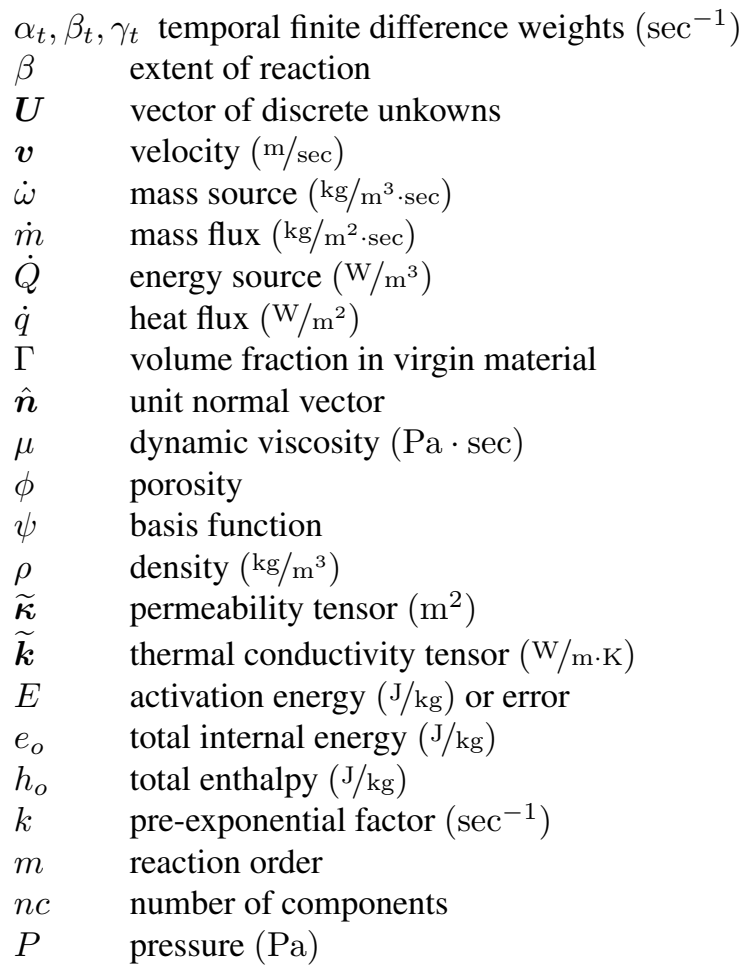

\footnotetext{
*Applied Aeroscience and CFD Branch, Member AIAA.

${ }^{\dagger}$ Applied Aeroscience and CFD Branch, Member AIAA.
} 
$p \quad$ temporal order of accuracy

$R \quad$ gas constant $(\mathrm{J} / \mathrm{kg} \cdot \mathrm{K})$

$T$ temperature $(\mathrm{K})$

$t$ time (sec)

$v \quad$ test function

\section{Subscripts and Superscripts}

$c \quad$ quantity of the char

$g \quad$ quantity of the gas

$h$ denotes finite dimensional approximation

$i \quad$ component index

$m \quad$ quantity of the mesh

$n \quad$ time index

$v \quad$ quantity of the virgin

\section{Introduction}

CHAR's primary purpose is to perform engineering analysis for design work. Provide brief literature review. Provide snapshot of paper.

\section{Governing Equations}

CHAR solves the governing equations for heat conduction and gas flow through decomposing porous media in one, two, or three dimensions. In general, this would include

- Continuity equations for each of the chemically reacting gaseous species resulting from flowfielnd injection and pyrolysis gas generation through in-depth decomposition

- Momentum equations accounting for porous media transport

- Energy equations for both the solid and gas phases

- Solid mass conservation equations for progression of pyrolysis

However, CHAR employs several simplifying assumptions to reduce the equation set to be more amenable for engineering application and design analyses. These assumptions include

- Flowfield gases are not allowed to penetrate the surface. Consequently, all the gas inside the porouse medium is generated through pryolysis.

- Pyrolysis gases do not react with or condense on the porous material.

- Pyrolysis gas diffusion is negelected such that the elemental composition of the gas is constant.

- All ablation occurs at the surface, and volume ablation effects are neglected.

- The solid and gas are assumed to be at the same temperature.

- The gas is assumed to be a mixture of perfect gases in chemical equilibrium.

- The momentum equations can be sufficiently represented by the steady form of Darcy's law.

Under these assumptions, the governing equations for a moving mesh simplify to

$$
\begin{aligned}
\text { Energy: } & \left.\frac{\partial\left(\rho e_{o}\right)}{\partial t}\right|_{\text {node }}-\nabla \cdot(\widetilde{\boldsymbol{k}} \boldsymbol{\nabla} T)+\boldsymbol{\nabla} \cdot\left(\phi \rho_{g} h_{o_{g}} \boldsymbol{v}_{g}\right)-\boldsymbol{v}_{m} \cdot \nabla\left(\rho e_{o}\right)-\dot{Q}=0 \\
\text { Gas Mass: } & \left.\frac{\partial\left(\phi \rho_{g}\right)}{\partial t}\right|_{\text {node }}-\dot{\omega}_{g}+\nabla \cdot\left(\phi \rho_{g} \boldsymbol{v}_{g}\right)-\boldsymbol{v}_{m} \cdot \nabla\left(\phi \rho_{g}\right)=0
\end{aligned}
$$


where the node subscript denotes conservation with respect to a moving node and Darcy's law defines the velocity field by

$$
\boldsymbol{v}_{g}=-\frac{\widetilde{\boldsymbol{\kappa}}}{\phi \mu} \nabla P
$$

Additionally, the solid mass conservation equation, given by

$$
\frac{\partial \rho_{s}}{\partial t}=\dot{\omega}_{s}
$$

is solved on a fixed mesh and treated as a constitutive relation when defining the temporal derivative in the energy equation.

\section{Numerical Methods}

\section{III.A. Finite Element Formulation}

In order to develop the finite element equations, a Galerkin weak statement can be developed for the energy equation in Eqn. (1), by first multiplying it by a suitable test function, $v$, and integrating over the domain $\Omega$ while integrating the second and third terms by parts to give the natural boundary condition terms. The weak statement is then: Find $\rho e_{o} \in H^{1}$ such that

$$
\begin{aligned}
\int_{\Omega}\left[v \frac{\partial\left(\rho e_{o}\right)}{\partial t}+\nabla v \cdot(\tilde{\boldsymbol{k}} \boldsymbol{\nabla} T)-\boldsymbol{\nabla} v \cdot\left(\phi \rho_{g} h_{o_{g}} \boldsymbol{v}_{g}\right)-v \boldsymbol{v}_{m} \cdot \boldsymbol{\nabla}\left(\rho e_{o}\right)-v \dot{Q}\right] d \Omega & \\
& +\oint_{\Gamma}\left(v h_{o_{g}} \dot{m}_{g}+v \dot{q}_{c o n d_{s}}\right) d \Gamma=0 \quad \forall v \in H_{0}^{1}
\end{aligned}
$$

where the boundary mass flux due to gas convection is

$$
\dot{m}_{g}=\left(\phi \rho_{g}\right) \boldsymbol{v}_{g} \cdot \hat{\boldsymbol{n}}
$$

and the boundary heat flux is

$$
\dot{q}_{\text {cond }_{s}}=-\tilde{\boldsymbol{k}} \boldsymbol{\nabla} T \cdot \hat{\boldsymbol{n}}
$$

Likewise, a Galerkin weak statement can be developed for the gas mass conservation equation in Eqn. (1). Again, the equation will be multiplied by a suitable test function, $v$, and integrated over the domain $\Omega$ while integrating the third term by parts to give the natural boundary condition term. The weak statement is then: Find $\phi \rho_{g} \in H^{1}$ such that

$$
\int_{\Omega}\left(\frac{\partial\left(\phi \rho_{g}\right)}{\partial t} v-\nabla v \cdot\left(\phi \rho_{g} \boldsymbol{v}_{g}\right)-v \boldsymbol{v}_{m} \cdot \nabla\left(\phi \rho_{g}\right)+\dot{\omega}_{s} v\right) d \Omega+\oint_{\Gamma} v \dot{m}_{g} d \Gamma=0 \quad \forall v \in H_{0}^{1}
$$

Eqns. (4) and (7) can be discretized by expanding the independent variables and test functions in terms of a finite dimensional basis

$$
\begin{aligned}
\left(\rho e_{o}\right)_{h}(\boldsymbol{x}) & =\sum_{j=1}^{\# \text { nodes }}\left(\rho e_{o}\right)_{j} \psi_{j}(\boldsymbol{x}) \\
\left(\phi \rho_{g}\right)_{h}(\boldsymbol{x}) & =\sum_{j=1}^{\# \text { nodes }}\left(\phi \rho_{g}\right)_{j} \psi_{j}(\boldsymbol{x}) \\
T_{h}(\boldsymbol{x}) & =\sum_{j=1}^{\# \text { nodes }} T_{j} \psi_{j}(\boldsymbol{x}) \\
P_{h}(\boldsymbol{x}) & =\sum_{j=1}^{\# \text { nodes }} P_{j} \psi_{j}(\boldsymbol{x}) \\
v_{h}(\boldsymbol{x}) & =\sum_{i=1}^{\# \text { nodes }} v_{i} \psi_{i}(\boldsymbol{x})
\end{aligned}
$$


where the subscript $h$ is introduced to denote a finite dimensional approximation. The unknowns in the system are chosen to be the nodal temperatures and pressure, $T_{j}$ and $P_{j}$. Since the unknowns are not functions of $\boldsymbol{x}$, the partial differential equation system is reduced to an ordinary differential equation system in which the temporal derivatives can be defined as

$$
\begin{aligned}
\frac{\partial\left(\rho e_{o}\right)_{h}}{\partial t} & =\sum_{j=1}^{\# \text { nodes }} \frac{d\left(\rho e_{o}\right)_{j}}{d t} \psi_{j}(\boldsymbol{x}) \\
\frac{\partial\left(\phi \rho_{g}\right)_{h}}{\partial t} & =\sum_{j=1}^{\# \text { nodes }} \frac{d\left(\phi \rho_{g}\right)_{j}}{d t} \psi_{j}(\boldsymbol{x})
\end{aligned}
$$

Since the equation system should be satisfied for all combinations of nodal shape function coefficients, $v_{i}$, their choice is arbitrary as long as a unique combination is chosen for each node. In typical finite element fashion, the coefficients are chosen to be $v_{i}=\delta_{i l}$ for the $l^{\text {th }}$ nodal equation. Consequently, the nodal residual equations resulting from Eqns. (4) and (7) can now be written as

$$
\begin{array}{rl}
\int_{\Omega} \psi_{i} \frac{\partial\left(\rho e_{o}\right)_{h}}{\partial t} d \Omega+\int_{\Omega} \boldsymbol{\nabla} \psi_{i} & \boldsymbol{k} \boldsymbol{\nabla} T_{h} d \Omega-\int_{\Omega}\left(\phi \rho_{g}\right)_{h} h_{o_{g}} \nabla \psi_{i} \cdot \boldsymbol{v}_{g} d \Omega \\
& -\int_{\Omega} \psi_{i} \boldsymbol{v}_{m} \cdot \nabla\left(\rho e_{o}\right)_{h} d \Omega+\oint_{\Gamma} \psi_{i} \dot{q}_{c o n d_{s}} d \Gamma+\oint_{\Gamma} \psi_{i} h_{o_{g}} \dot{m}_{g} d \Gamma-\int_{\Omega} \psi_{i} \dot{Q} d \Omega=0
\end{array}
$$

and

$$
\begin{aligned}
\int_{\Omega} \psi_{i} \frac{\partial\left(\phi \rho_{g}\right)_{h}}{\partial t} d \Omega-\int_{\Omega}\left(\phi \rho_{g}\right)_{h} \nabla \psi_{i} \cdot \boldsymbol{v}_{g} d \Omega-\int_{\Omega} \psi_{i} \boldsymbol{v}_{m} \cdot \nabla\left(\phi \rho_{g}\right)_{h} d \Omega & +\oint_{\Gamma} \psi_{i} \dot{m}_{g} d \Gamma+\int_{\Omega} \psi_{i} \dot{\omega}_{s} d \Omega=0
\end{aligned}
$$

for $i=1,2, \ldots, \#$ nodes.

\section{III.B. Time Discretization}

The semidiscrete weak form of the system given by Eqns. (15) and (16) is discretized in time using backward finitedifference schemes. Both first- and second-order accurate schemes may be derived from Taylor series expansions in time about $\boldsymbol{U}_{n+1}$ as outlined by $\mathrm{Kirk}^{1}$ to give

$$
\frac{\partial \boldsymbol{U}_{n+1}}{\partial t}=\alpha_{t} \boldsymbol{U}_{n+1}+\beta_{t} \boldsymbol{U}_{n}+\gamma_{t} \boldsymbol{U}_{n-1}+\mathcal{O}\left(\Delta t_{n+1}^{p}\right)
$$

where the weights are given for $p=1$ and $p=2$ in Table 1.

Table 1. First- and Second-Order Accurate Time Discretization Coefficients
\begin{tabular}{c||ccc}
$\boldsymbol{p}$ & $\boldsymbol{\alpha}_{\boldsymbol{t}}$ & $\boldsymbol{\beta}_{\boldsymbol{t}}$ & $\boldsymbol{\gamma}_{\boldsymbol{t}}$ \\
\hline \hline & & $\frac{-1}{\Delta t_{n+1}}$ & 0 \\
1 & $\frac{1}{\Delta t_{n+1}}$ & $-\left[\frac{1}{\Delta t_{n+1}}+\frac{1}{\Delta t_{n}}\right]$ & $\frac{\Delta t_{n+1}}{\Delta t_{n}\left(\Delta t_{n+1}+\Delta t_{n}\right)}$ \\
2 & $-\beta_{t}-\gamma_{t}$ & $-\left[\begin{array}{l} \\
\end{array}\right.$
\end{tabular}

The resulting temporal derivatives are given by

$$
\frac{d\left(\rho e_{o}\right)_{j}}{d t}=\alpha_{t}\left(\rho e_{o}\right)_{j}^{n+1}+\beta_{t}\left(\rho e_{o}\right)_{j}^{n}+\gamma_{t}\left(\rho e_{o}\right)_{j}^{n-1}
$$

and

$$
\frac{d\left(\phi \rho_{g}\right)_{j}}{d t}=\alpha_{t}\left(\phi \rho_{g}\right)_{j}^{n+1}+\beta_{t}\left(\phi \rho_{g}\right)_{j}^{n}+\gamma_{t}\left(\phi \rho_{g}\right)_{j}^{n-1}
$$

which can be substituted into Eqns. (13) and (14) respectively. 


\section{Material Model}

The material model is an extension of the CMA model developed by Moyer and Rindal. ${ }^{2}$ It is assumed that all the pores are interconnected, and therefore pyrolysis gases occupy all of the pore space and are free to flow through it. Consequently, the density of the solid/gas system is described by

$$
\rho=\phi \rho_{g}+\rho_{s}
$$

where the solid density is a bulk density, the gas density is with respect to the space the gas occupies (pore space), and the porosity is equal to the gas volume fraction. In terms of units, Eqn. (20) can be expressed as

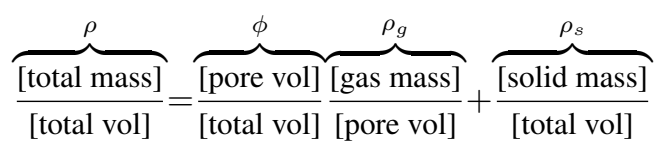

The solid material is modelled as a mixture of components each with their own rate law governing its decomposition.

$$
\rho_{s}=\sum_{i=1}^{n c} \Gamma_{i} \rho_{s_{i}}
$$

The units associated with the solid bulk density model in Eqn. (22) are

$$
\overbrace{\frac{\text { [solid mass }]}{[\text { total vol] }}}^{\rho_{s}}=\sum_{i=1}^{n c} \overbrace{\frac{\left[\text { initial vol of } i^{t h} \text { comp. }\right]}{[\text { total vol }]}}^{\Gamma_{i}} \overbrace{\left[\text { [mass of } i^{t h} \text { comp. }\right]}^{\rho_{i}}
$$

It is assumed that the solid does not change volume due to thermal expansion, and therefore the total volume is constant. It is important to note that the solid description in Eqn. (22) is only a modeling assumption, and the solid is not truly comprised of $n c$ components, species, or distinguishable materials. Taking the temporal derivative of Eqn. (22) gives the solid decomposition rate in terms of component decomposition rates.

$$
\frac{\partial \rho_{s}}{\partial t}=\dot{\omega}_{s}=\sum_{i=1}^{n c} \Gamma_{i} \frac{\partial \rho_{s_{i}}}{\partial t}=\sum_{i=1}^{n c} \Gamma_{i} \dot{\omega}_{s_{i}}
$$

It is assumed that the decomposition of each component can be described by an Arrhenius relationship of the form

$$
\dot{\omega}_{s_{i}}=-k_{i} \rho_{v_{i}}\left(\frac{\rho_{i}-\rho_{c_{i}}}{\rho_{v_{i}}}\right)^{\psi_{i}} e^{-E_{i} / R T}
$$

which applies at a constant spatial location (as apposed to a given node that can move during the solution process). The decomposition kinetics in Eqn. (25) are simplified to ordinary differential equations and integrated as part of the solution of the energy equation.

Since most thermophysical properties of the solid are only known for the virgin and fully charred states, the intermediate solid is modeled as some interpolated state between virgin and char. This interpolated state is characterized by the extent of reaction $(\beta)$, or degree of char, given by

$$
\beta=\frac{\rho_{v}-\rho_{s}}{\rho_{v}-\rho_{c}}
$$

where the virgin and char bulk densities are known constants. The definition in Eqn. (26) can be rearranged to more clearly describe the interpolated state.

$$
\rho_{s}=(1-\beta) \rho_{v}+\beta \rho_{c}
$$

Although the virgin and char materials are not distinguishable entities within the intermediate solid, Eqn. (27) reveals that the degree of char represents an effective char volume fraction within the solid (not in the solid/gas mixture). In a similar light, CMA defines an effective virgin mass fraction given by

$$
y_{v}=\frac{\rho_{v}}{\rho_{v}-\rho_{c}}\left(1-\frac{\rho_{c}}{\rho_{s}}\right)
$$


which can be related to the extent of reaction through

$$
y_{v}=\frac{\rho_{v}}{\rho_{s}}(1-\beta)
$$

Similarly the char mass fraction is given by

$$
y_{c}=1-y_{v}=\frac{\rho_{c}}{\rho_{s}} \beta
$$

These effective parameters are used to interpolate between virgin and char properties for partially decomposed material.

\section{Code Architecture}

To expedite the software development process, the development team has chosen to take advantage of pre-existing, high quality, and freely available software libraries to avoid duplicating the efforts of others. Consequently, the CHAR program can be thought of as an ablation and thermal physics wrapper around a hierarchy of libraries that aid in the solution and data management processes. The primary software packages included in CHAR are outlined in Figure 1, and there are additional libraries that the noted libraries rely on for the functionality in CHAR. The majority of the physics and application-dependent code is in the top-level routines denoted by the CHAR box. This includes simulation management, initialization, calls to library functions, matrix assembly routines for element-level integration, boundary condition application, and material property data structures and look-up routines. Those routines comprised the bulk of the development effort for the current study.

Eigen $^{3}$ is a library for linear algebra including: matrix/vector math, numerical solvers, and other related algorithms. It is primarily used in CHAR for its matrix and vector data structures and solutions to small linear systems

Cantera ${ }^{4}$ is a collection of object-oriented software tools for problems involving chemical kinetics, thermodynamics, and transport processes. For CHAR, it is used to calculate thermodynamic state properties and transport properties for gaseous mixtures in equilibrium. It is used for both the in-depth gas mixture and the environment gas mixture at surface conditions. Since repetitive calls to the surface equilibrium calculator can get expensive over the coarse of a simulation, Cantera is only used in the beginning of a simulation to build the required data tables over a range of relevant conditions for the problem. Subsequently, CHAR interpolates in these data tables to determine gas properties.

The workhorse for CHAR is the libMesh finite-element library, which is developed at NASA Johnson Space Center and the University of Texas at Austin with many other contributors around the world. libMesh performs nearly all of the physics independent tasks of the software and can be thought of as the framework on which the physics-dependent application code is built. While the functions of libMesh within CHAR are many, the notable functions include linear system data management, mesh data management, parallel communication, finite-element utilities that streamline integration, and adaptive mesh refinement. libMesh also provides user-transparent interfaces to the Portable, Extensible Toolkit for Scientific Comuptation (PETSc) library, ${ }^{5}$ which solves the assembled linear system, and METIS, ${ }^{6}$ which provides the domain decomposition for parallel execution.

\section{Boundary Conditions}

\section{VI.A. Thermochemical Ablation}

VI.B. Surface-to-Surface Radiation Exchange

VI.C. Flowfield Coupling

\section{Mesh Motion}

\section{Adaptive Mesh Refinement}

\section{Inverse Solver}

\section{Thermoelastic Solver}

CHAR has the ability to solve the linear thermoelastic equations in a one-way coupled quasi-steady fashion to give the displacement, stress, and strain fields for a body under thermoelastic loading. The equations are linearized by 


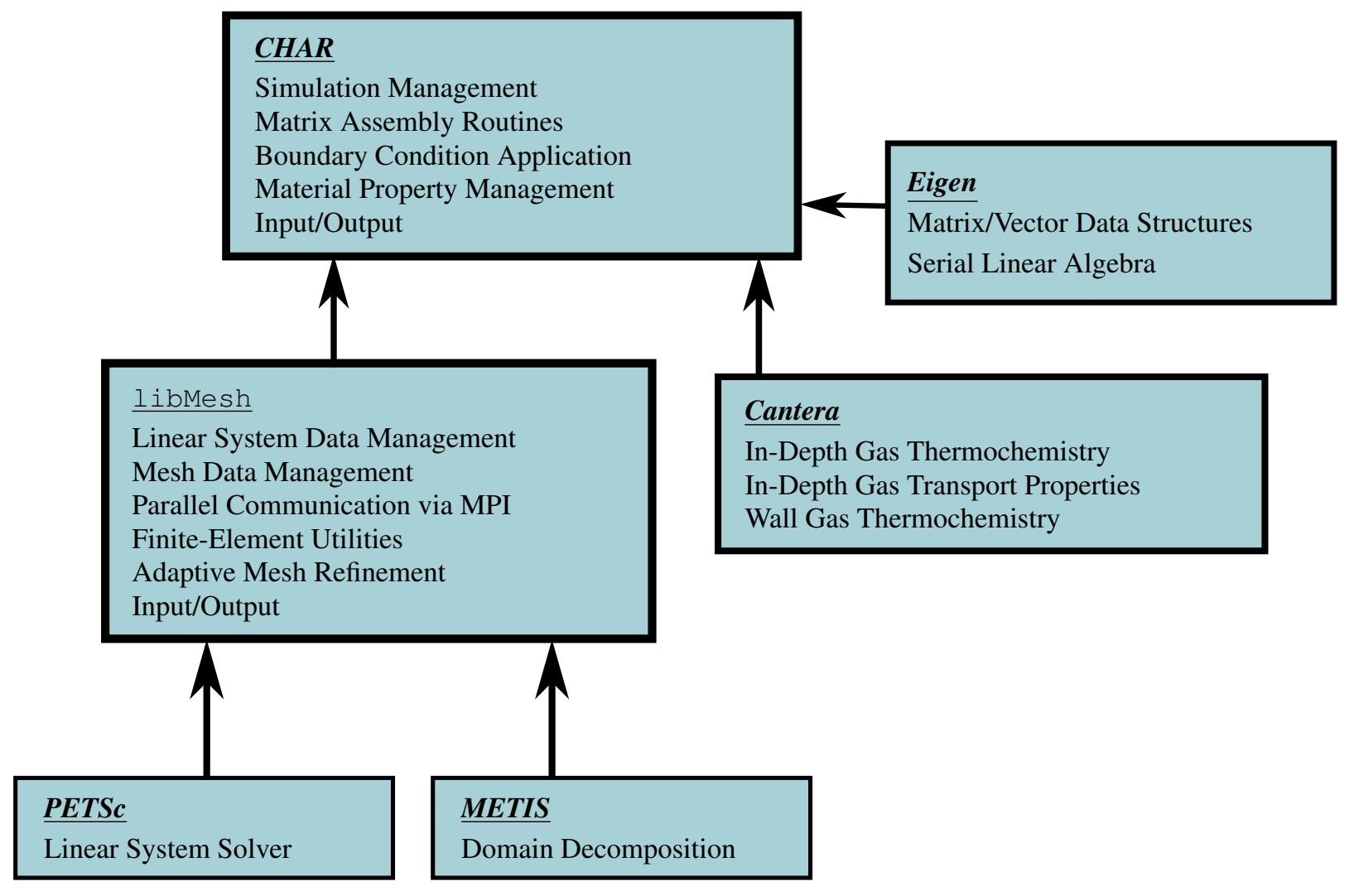

Figure 1. CHAR software architecture. 
only considering the linear terms in the strain-displacement relationships and by assuming that the material properties are not functions of the displacement. The thermoelastic equations are one-way coupled to the thermal and ablation equations such that an instantaneous temperature field provided from the thermal and ablation solver is assumed to be fixed during the thermoelastic solve. There is no feedback from the thermoelastic solver to the energy equation either in the form of solid deformation or thermoelastic coupling terms in the energy equation. The thermoelastic solver can be thought of as a post-processing of the thermal and ablation equations that can occur at a user-defined frequency. In the current implementation, the pressure field within a decomposing ablator is not considered to contribute to the thermoelastic response. It is recognized that this capability must be added to predict internal pressure induced spallation.

The solution to the thermoelastic equations is quasi-steady in that the displacement field, $\boldsymbol{u}$, is assumed to be static at the end of each solve. This implies that

$$
\frac{\partial \boldsymbol{u}}{\partial t}=\mathbf{0}
$$

The physical assumption is that the material elastically responds infinitely fast relative to changes in the temperature field.

\section{Governing Equations}

The equations that govern the steady thermoelastic response of a solid under loading are

$$
\nabla \cdot \sigma+f=0
$$

where $\boldsymbol{\sigma}$ is the stress tensor, $\boldsymbol{f}$ is the body force per unit volume, $\rho$ is the material's density, and $\boldsymbol{u}$ is the displacement field.

$$
\boldsymbol{u}=\left[\begin{array}{l}
u_{1} \\
u_{2} \\
u_{3}
\end{array}\right]
$$

The symmetric stress tensor is defined as

$$
\boldsymbol{\sigma}=\left[\begin{array}{ccc}
\sigma_{11} & \sigma_{12} & \sigma_{13} \\
\cdot & \sigma_{22} & \sigma_{23} \\
\cdot & \cdot & \sigma_{33}
\end{array}\right]=\left[\begin{array}{lll}
\boldsymbol{\sigma}_{1} & \boldsymbol{\sigma}_{2} & \boldsymbol{\sigma}_{3}
\end{array}\right]
$$

where the diagonal terms are the normal stresses and the off-diagonal terms are the shear stresses. where

$$
\boldsymbol{\sigma}_{d}=\left[\begin{array}{c}
\sigma_{1 d} \\
\sigma_{2 d} \\
\sigma_{3 d}
\end{array}\right] \quad \text { for } d=1,2,3
$$

The linear elasticity equation for each dimension can now be written as

$$
\boldsymbol{\nabla} \cdot \boldsymbol{\sigma}_{d}+f_{d}=0 \quad \text { for } d=1,2,3
$$

In order to derive the relationship between the stress and the displacements, it is necessary to recognize that the orthogonal 123-coordinate system that the problem is solved in (denoted as the "problem coordinate system" from here on) is in general not the same orthogonal $1^{\prime} 2^{\prime} 3^{\prime}$-coordinate system that the material properties are defined in (denoted as the "material coordinate system" from here on). Any value, vector, or tensor followed by the prime symbol, "', will be with respect to the material coordinate system, while any non-primed value, vector, or tensor will be with respect to the problem coordinate system.

In the material coordinate system, the stress is related to the strain via Hooke's law for small deformations

$$
\boldsymbol{\sigma}^{\prime}=\boldsymbol{K}^{\prime}:\left[\boldsymbol{\epsilon}^{\prime}-\Delta T \boldsymbol{\alpha}^{\prime}\right]
$$

where the strain, $\boldsymbol{\epsilon}^{\prime}$, is also a symmetric tensor and is defined via the linear strain-displacement relationship

$$
\boldsymbol{\epsilon}^{\prime}=\frac{1}{2}\left[\boldsymbol{\nabla} \boldsymbol{u}^{\prime}+\left(\nabla \boldsymbol{u}^{\prime}\right)^{T}\right]
$$


$\Delta T \boldsymbol{\alpha}^{\prime}$ are the thermal strains where

$$
\boldsymbol{\alpha}^{\prime}=\left[\begin{array}{ccc}
\alpha_{1}^{\prime} & 0 & 0 \\
\cdot & \alpha_{2}^{\prime} & 0 \\
\cdot & \cdot & \alpha_{3}^{\prime}
\end{array}\right]
$$

and $\alpha_{i}^{\prime}$ are the coefficients of thermal expansion. $\boldsymbol{K}^{\prime}$ is the fourth-order stiffness tensor containing the 81 elastic coefficients, $k_{i j k l}^{\prime}$, which are properties of the material and are defined with respect to the material's coordinate system. Because of the linear assumption, the elastic coefficients are independent of the displacement, but they do vary with temperature. Due to symmetry, there are only 21 independent elastic coefficients in the stiffness tensor. Consequently, Voigt notation is often used to represent the stress and strain tensors to reduce the order of the problem.

$$
\boldsymbol{\sigma}^{\prime}=\left[\begin{array}{c}
\sigma_{11}^{\prime} \\
\sigma_{22}^{\prime} \\
\sigma_{33}^{\prime} \\
\sigma_{23}^{\prime} \\
\sigma_{31}^{\prime} \\
\sigma_{12}^{\prime}
\end{array}\right], \boldsymbol{\epsilon}^{\prime}=\left[\begin{array}{c}
\epsilon_{11}^{\prime} \\
\epsilon_{22}^{\prime} \\
\epsilon_{33}^{\prime} \\
2 \epsilon_{23}^{\prime} \\
2 \epsilon_{31}^{\prime} \\
2 \epsilon_{12}^{\prime}
\end{array}\right], \boldsymbol{\alpha}^{\prime}=\left[\begin{array}{c}
\alpha_{1}^{\prime} \\
\alpha_{2}^{\prime} \\
\alpha_{3}^{\prime} \\
0 \\
0 \\
0
\end{array}\right]
$$

and the stiffness tensor is simplified to

$$
\boldsymbol{K}_{r}^{\prime}=\left[\begin{array}{llllll}
k_{1111}^{\prime} & k_{1122}^{\prime} & k_{1133}^{\prime} & k_{1123}^{\prime} & k_{1131}^{\prime} & k_{1112}^{\prime} \\
k_{2211}^{\prime} & k_{2222}^{\prime} & k_{2233}^{\prime} & k_{2223}^{\prime} & k_{2231}^{\prime} & k_{2212}^{\prime} \\
k_{3311}^{\prime} & k_{3322}^{\prime} & k_{3333}^{\prime} & k_{3323}^{\prime} & k_{3331}^{\prime} & k_{3312}^{\prime} \\
k_{2311}^{\prime} & k_{2322}^{\prime} & k_{2333}^{\prime} & k_{2323}^{\prime} & k_{2331}^{\prime} & k_{2312}^{\prime} \\
k_{3111}^{\prime} & k_{3122}^{\prime} & k_{3133}^{\prime} & k_{3123}^{\prime} & k_{3131}^{\prime} & k_{3112}^{\prime} \\
k_{1211}^{\prime} & k_{1222}^{\prime} & k_{1233}^{\prime} & k_{1223}^{\prime} & k_{1231}^{\prime} & k_{1212}^{\prime}
\end{array}\right]=\left[\begin{array}{cccccc}
K_{11}^{\prime} & K_{12}^{\prime} & K_{13}^{\prime} & K_{14}^{\prime} & K_{15}^{\prime} & K_{16}^{\prime} \\
\cdot & K_{22}^{\prime} & K_{23} & K_{24} & K_{25} & K_{26} \\
\cdot & \cdot & K_{33}^{\prime} & K_{34}^{\prime} & K_{35}^{\prime} & K_{36}^{\prime} \\
\cdot & \cdot & \cdot & K_{44}^{\prime} & K_{45}^{\prime} & K_{46}^{\prime} \\
\cdot & \cdot & \cdot & \cdot & K_{55}^{\prime} & K_{56}^{\prime} \\
\cdot & \cdot & \cdot & . & \cdot & K_{66}^{\prime}
\end{array}\right]
$$

where the subscript $r$ is introduced to differentiate the reduced order tensor from the original $4^{\text {th }}$ order stiffness tensor, $\boldsymbol{K}^{\prime}$. With this new notation, the stress can be calculated according to

$$
\boldsymbol{\sigma}^{\prime}=\boldsymbol{K}_{r}^{\prime}\left[\boldsymbol{\epsilon}^{\prime}-\Delta T \boldsymbol{\alpha}^{\prime}\right]
$$

or

$$
\left[\begin{array}{c}
\sigma_{11}^{\prime} \\
\sigma_{22}^{\prime} \\
\sigma_{33}^{\prime} \\
\sigma_{23}^{\prime} \\
\sigma_{13^{\prime}} \\
\sigma_{12}^{\prime}
\end{array}\right]=\left[\begin{array}{cccccc}
K_{11}^{\prime} & K_{12}^{\prime} & K_{13}^{\prime} & K_{14}^{\prime} & K_{15}^{\prime} & K_{16}^{\prime} \\
\cdot & K_{22}^{\prime} & K_{23} & K_{24} & K_{25} & K_{26} \\
\cdot & \cdot & K_{33}^{\prime} & K_{34}^{\prime} & K_{35}^{\prime} & K_{36}^{\prime} \\
\cdot & \cdot & \cdot & K_{44}^{\prime} & K_{45}^{\prime} & K_{46}^{\prime} \\
\cdot & \cdot & \cdot & \cdot & K_{55}^{\prime} & K_{56}^{\prime} \\
\cdot & \cdot & \cdot & \cdot & \cdot & K_{66}^{\prime}
\end{array}\right]\left(\left[\begin{array}{c}
\epsilon_{11}^{\prime} \\
\epsilon_{22}^{\prime} \\
\epsilon_{33}^{\prime} \\
2 \epsilon_{23}^{\prime} \\
2 \epsilon_{31}^{\prime} \\
2 \epsilon_{12}^{\prime}
\end{array}\right]-\Delta T\left[\begin{array}{c}
\alpha_{1}^{\prime} \\
\alpha_{2}^{\prime} \\
\alpha_{3}^{\prime} \\
0 \\
0 \\
0
\end{array}\right]\right)
$$

The stress vector, $\sigma^{\prime}$, can now be used to fill out the stress tensor, $\boldsymbol{\sigma}^{\prime}$. Next, a rotation matrix, $\boldsymbol{C}$, is required to get the stress tensor from the material coordinate system to the problem coordinate system.

$$
\sigma=C^{T} \sigma^{\prime} C
$$

where $C$ is defined in Section ??.

In CHAR, the approach will be to use Eqns. (42) and (44) to define the stress tensor for integration of the PDEs. The strain vector in Eqn. (42) is defined using the displacements in the material coordinate system, $\boldsymbol{u}^{\prime}$. Since the unknowns are defined in the problem coordinate system, it is more convenient to work in the problem coordinate system when defining the strain vector. Consequently, the strain vector in Eqn. (42) will be calculated by rotating the strain tensor from the problem coordinate system to the material coordinate system.

$$
\epsilon^{\prime} \Leftarrow \epsilon^{\prime}=C^{T^{-1}} \epsilon C^{-1}
$$

where the $\Leftarrow$ symbol denotes filling in the strain vector given the values in the strain tensor. Since $C$ is an orthogonal matrix

$$
C^{-1}=C^{T}
$$

and consequently

$$
\epsilon^{\prime} \Leftarrow \epsilon^{\prime}=C \epsilon C^{T}
$$




\section{Finite Element Formulation}

The Galerkin weak statement for the equations can be developed using Eqn. (36). The equation is multiplied by a suitable test function, $v$, and integrated over the domain, $\Omega$, while integrating the first term by parts to give the natural boundary condition term. The weak statement is then: Find $\boldsymbol{u} \in H^{1}$ such that

$$
\int_{\Omega} \boldsymbol{\nabla} v \cdot \boldsymbol{\sigma}_{d} d \Omega-\int_{\Omega} v f_{d} d \Omega-\int_{\Gamma} v\left(\boldsymbol{\sigma}_{d} \cdot \hat{\boldsymbol{n}}\right) d \Gamma=0 \quad \forall v \in H_{0}^{1} \quad \text { for } d=1,2,3
$$

Eqn. (48) can be discretized by expanding the independent variables and test functions in terms of a finite dimensional basis

$$
\begin{aligned}
\left(u_{d}\right)_{h}(\boldsymbol{x}) & =\sum_{j=1}^{\text {\# nodes }}\left(u_{d}\right)_{j} \psi_{j}(\boldsymbol{x}) \\
\boldsymbol{\nabla}\left(u_{d}\right)_{h}(\boldsymbol{x}) & =\sum_{j=1}^{\text {\#nodes }}\left(u_{d}\right)_{j} \boldsymbol{\nabla} \psi_{j}(\boldsymbol{x}) \\
v_{h}(\boldsymbol{x}) & =\sum_{i=1}^{\text {\# nodes }} v_{i} \psi_{i}(\boldsymbol{x}) \\
\boldsymbol{\nabla} v_{h}(\boldsymbol{x}) & =\sum_{i=1}^{\text {\#nodes }} v_{i} \boldsymbol{\nabla} \psi_{i}(\boldsymbol{x})
\end{aligned}
$$

where the subscript $h$ is introduced to denote a finite dimensional approximation. Now, the unknowns are no longer functions of $\boldsymbol{x}$. Since the equation system should be satisfied for all combinations of nodal test function coefficients, $v_{i}$, their choice is arbitrary. Choosing a unique combination for each node allows for the development of a linear system where the number of equations equals the number of unknowns. In typical finite element fashion, the coefficients are chosen to be $v_{i}=\delta_{i l}$ for the $l^{t h}$ nodal equation. Consequently the nodal residual equations can now be written as

$$
\int_{\Omega} \nabla \psi_{i} \cdot \boldsymbol{\sigma}_{d} d \Omega-\int_{\Omega} \psi_{i} f_{d} d \Omega-\int_{\Gamma} \psi_{i} T_{d} d \Gamma=0 \quad \text { for } d=1,2,3
$$

for $i=1,2, \ldots, \#$ nodes. The surface traction is defined as

$$
T_{d}=\boldsymbol{\sigma}_{d} \cdot \hat{\boldsymbol{n}} \quad \text { for } d=1,2,3
$$

\section{Ongoing Development Efforts}

- Water phase change model

- Unsteady porous flow momentum equations

- Thermal non-equilibrium between solid and gas

- Chemical non-equilibrium modeling

\section{Conclusions}

\section{Acknowledgments}

Ben Blackwell, Micah Howard, Nathan Calvert

\section{References}

${ }^{1}$ Benjamin S. Kirk. Adaptive Finite Element Simulation of Flow and Transport Applications on Parallel Computers. $\mathrm{PhD}$ thesis, The University of Texas at Austin, May 2007. 
${ }^{2}$ Carl B. Moyer and Roald A. Rindal. An Analysis of the Coupled Chemically Reacting Boundary Layer and Charring Ablator, Part II: Finite Difference Solution for the In-Depth Response of Charring Materials Considering Surface Chemical and Energy Balances. Technical Report 66-7 Part II, Aerotherm, March 1967.

3 Gaël Guennebaud, Benoît Jacob, et al. Eigen v3. http://eigen.tuxfamily.org, 2010.

4 David G. Goodwin. Cantera c++ user's guide. Technical report, California Institute of Technology, October 2002.

${ }^{5}$ Satish Balay, Kris Buschelman, Victor Eijkhout, William D. Gropp, Dinesh Kaushik, Matthew G. Knepley, Lois Curfman McInnes, Barry F. Smith, and Hong Zhang. PETSc users manual. Technical Report ANL-95/11 - Revision 2.3.0, Argonne National Laboratory, April 2004.

${ }^{6}$ G. Karypis and V. Kumar. METIS unstructured graph partitioning and sparse matrix order. Technical report, University of Minnesota, Department of Computer Science, August 1995. 